\title{
Helicobacter, Inflammation, and Gastric Cancer
}

\author{
Antonia R. Sepulveda
}

Published online: 2 February 2013

(c) Springer Science+Business Media New York 2013

\begin{abstract}
Helicobacter pylori infection leads to longlasting chronic inflammation and represents the most common risk factor underlying gastric cancer. Recently, new insights into the mechanisms through which $H$. pylori and mucosal inflammation lead to cancer development have emerged. $H$. pylori virulence factors, in particular specific $C a g A$ genotypes, represent main factors in gastric cancer, inducing altered intracellular signaling in epithelial cells. The chronic nature of $H$. pylori infection appears to relate to the VacA virulence factor and Th17/Treg mechanisms. A role of $H$. pylori infection in epigenetic and microRNA deregulation has been shown. Mutations of the epithelial cell genome, a hallmark of cancer, were demonstrated to accumulate in $H$. pylori infected stomach partly due to inadequate DNA repair. Gastric stem cells were shown to be targets of oxidative injury in the Helicobacter-inflammatory milieu. Recent advances emphasizing the contribution of bacterial factors, inflammatory mediators, and the host epithelial response in gastric carcinogenesis are reviewed.
\end{abstract}

Keywords Helicobacter - H. pylori - Inflammation · Mutation · DNA repair - Gastric cancer - Stem cells · Pathobiology

\section{Introduction}

Gastric cancer (GC) is the most frequent malignancy arising in the stomach and represents the fourth most

\footnotetext{
A. R. Sepulveda $(\bowtie)$

Department of Pathology \& Cell Biology, Columbia University, West 168 Street, VC14-212, New York, NY 10030, USA

e-mail: as4400@columbia.edu
}

frequent cancer worldwide [1]. Helicobacter pylori (H. pylori) bacteria were identified as the main agent of chronic gastritis and ulcers by Warren and Marshal [2], and later studies revealed an association with GC [3], leading to the classification of $H$. pylori as a human carcinogen [4]. The attributable risk of GC related to $H$. pylori infection in the population has been estimated to be $75 \%$ [3].

Gastric cancers can be divided into distinct subtypes based on differential mechanisms of neoplastic initiation and underlying risk factors (inherited vs. sporadic), histopathologic, and molecular phenotypes. Sporadic type adenocarcinomas represent approximately $80 \%$ of all cases of $\mathrm{GC}$ and are associated with multifactorial causal factors, among which $H$. pylori chronic gastritis represents a main driving factor. Additionally, other factors known to play a role in GC development include host genetic susceptibility and carcinogens present in specific diets or smoking that work together with other factors such as the cellular injury caused by chronic gastritis to enhance the risk of GC $[5,6]$. The molecular subtypes of GC include tumors characterized by the chromosomal instability pathway (CIN), the most frequent molecular type of GC, while a smaller proportion of tumors develop through the microsatellite instability pathway (MSI), or the $\mathrm{CpG}$ island methylator phenotype pathway [7].

Gastric cancers are among the class of cancers that arise in association with chronic inflammation (chronic gastritis), as do other cancers in the gastrointestinal tract such as esophageal adenocarcinoma arising in association with reflux esophagitis and Barrett's esophagus, and colon cancers that arise in patients with inflammatory bowel diseases (ulcerative colitis and Crohn's disease). Although inflammation is a common factor in all these cancers, unique mechanisms that relate to specific factors involved in each type of cancer likely provide cancer signatures that 
may reflect the different underlying factors. To this end, in H. pylori associated GC, although inflammation is a major driver of neoplastic development, additional Helicobacterspecific induced alterations contribute to the landscape of molecular changes that may be unique to the H. pyloricancer association. This notion is supported by studies showing that $H$. pylori bacteria affect epithelial cell regulation in the absence of inflammatory products as demonstrated in many co-culture studies, and the bacterium virulence factors, in particular the CagA protein, have been shown to induce a unique and complex array of cellular signaling alterations [8] (Fig. 1).

\section{Role of $\boldsymbol{H}$. pylori in Gastric Carcinogenesis: Patterns of Host Response}

The chronic nature of $H$. pylori gastritis is critical to the carcinogenic potential of this infection, resulting in a longterm interaction of the bacteria and inflammatory mediators with differentiated gastric epithelial cells and epithelium progenitor and stem cells, with accumulation of mutations, epigenetic modifications and deregulation of cell function that may ultimately lead to neoplasia. Therefore, $H$. pylori infection plays a critical role during the initiating steps of GC.

Helicobacter pylori infection is usually acquired during childhood, with ensuing chronic gastritis as the norm and various possible complications including gastric and duodenal ulcers, GC, and extranodal marginal zone B cell lymphoma of mucosa associated lymphoid tissue (MALTLymphoma) [9, 10]. Cure of $H$. pylori infection requires treatment with anti-H. pylori therapy [11]. Gastric cancer develops several decades after acquisition of the infection, following progressive mucosal damage secondary to continued interaction of $H$. pylori bacteria with the mucosa and the consequent chronic inflammatory milieu [12].

Histologically, H. pylori gastritis is characterized by a combination of chronic and acute inflammation of the gastric mucosa. There is progressive damage of gastric glands that leads to mucosal atrophy with intestinal metaplasia, overall resulting in a picture of atrophic gastritis, which constitutes an environment with enhanced risk of development of dysplasia and carcinoma. Extensive gastritis and mucosal atrophy in the gastric body and fundus lead to hypochlorhydria, which creates an environment that allows for overgrowth of other bacteria that may increase carcinogenic activity in the stomach through the conversion of nitrites to carcinogenic nitroso-N compounds [13]. Helicobacter pylori-associated pangastritis (involving both the body/fundus and antrum) is frequently seen in family relatives of GC patients, which may contribute to gastric cancer clustering in some families [14]. The histologic alterations that precede gastric cancer, including intestinal metaplasia, represent pre-neoplastic epithelial changes in gastric carcinogenesis and have been shown to carry numerous genomic, epigenetic and functional abnormalities that can also be detected in cancer tissues [6, 15-18]. Therefore, characterization of molecular alterations in the background mucosa before patients develop GC may offer an opportunity to identify patterns associated with increased risk of GC development.

The cancers arising in the inflammatory background of H. pylori-associated chronic gastritis are most commonly of intestinal type, which are predominantly well- to moderately-differentiated adenocarcinomas, but diffuse type adenocarcinomas, which are poorly cohesive and poorly differentiated and may include a variable component with
Fig. $1 H$. pylori virulence factors and inflammation mechanisms leading to gastric cancer

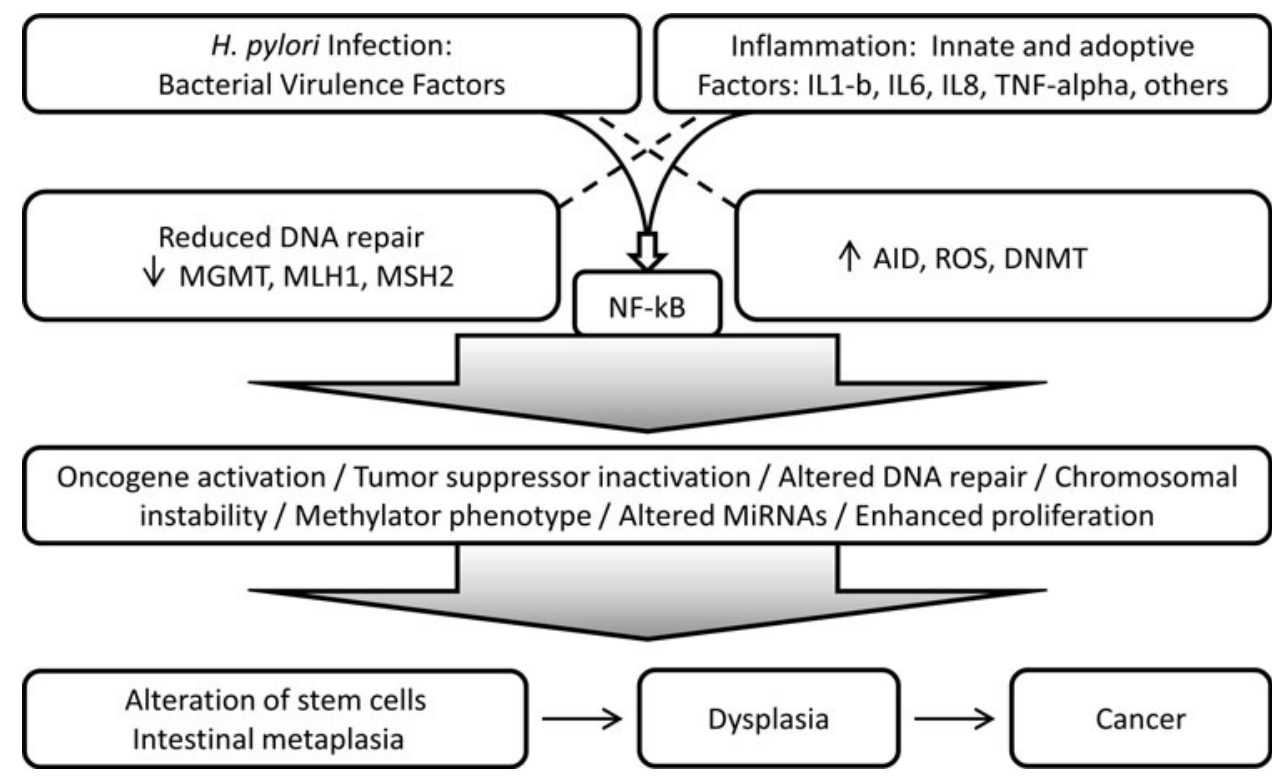


signet ring cell features, also occur in association with H. pylori $[19,20]$.

\section{H. pylori Infection and Inflammatory Host Response}

Helicobacter pylori infection of the stomach elicits both humoral and cellular immune responses [21]. The induced immunologic/inflammatory cascade reflects the activation of innate and acquired immune responses [21]. The characteristic long-term chronic inflammatory status of $H$. pylori infected gastric mucosa requires evasion of $H$. pylori from the immune system. The immune response to $H$. pylori is induced by bacterial products upon their contact with epithelial cells of gastric mucosa, and with macrophages and dendritic cells in the lamina propria that are reached after epithelial cells and intercellular junctions are damaged by $H$. pylori virulence factors such as the VacA toxin. Epithelial cells respond to $H$. pylori by undergoing cellular signaling changes and by releasing cytokines into the mucosal lamina propria, to activate macrophages, dendritic cells and other inflammatory cells. Inflammatory mediators released during H. pylori gastritis include interleukin IL-1, IL-6, IL-8, tumor necrosis factor (TNF)-alpha and regulated and normal $\mathrm{T}$ cell expressed and secreted (RANTES) [22]. Mediators released by macrophages, dendritic cells and epithelial cells activate T-lymphocytes with a predominant Th1 response [23], regulatory T-lymphocytes (Treg), B-lymphocytes which mature into mucosal plasma cells, and neutrophils which actively phagocytize $H$. pylori bacteria. In addition, there is a contribution of dentritic cells that release IL23 and activate the production of IL17 associated with a Th17 response against H. pylori [24]. However, studies indicate that $H$. pylori direct a Treg-skewed dendritic cell-induced helper $\mathrm{T}$ cell differentiation, in contrast to the Th17-skewed response seen with pro-inflammatory bacteria. The increased Treg induction in H. pylori infected-hosts forces an imbalance of the Th17/ Treg axis, which may lead to suppressed Th17 and ineffective bacterial eradication and persistence of $H$. pylori as a chronic infection [25•].

Helicobacter pylori stimulates the production of growth factors such as granulocyte-macrophage colony stimulating factors (GM-CSF) and inflammation regulators such as cyclo-oxygenase-2 (COX-2) and reactive oxygen/nitrogen species (ROS/RNS).

$\mathrm{NF}-\mathrm{kB}$ is a key regulator of inflammation and other cellular cascades that underlie carcinogenesis. NF-kB can be activated by numerous pro-inflammatory activators namely cytokines released by other cells and through Toll like receptor (TLR) activation by pathogen products, through two different pathways (the canonical and noncanonical pathways). Helicobacter pylori activation of $\mathrm{NF}-\mathrm{Kb}$ follows either the canonical or non-canonical pathways in epithelial cells and lymphoid cells, respectively [26]. Helicobacter pylori peptidoglycan is recognized by epithelial cells through the intracellular nucleotide binding and oligomerization domain 1 (NOD1), activating MAPKs in both the NF-kB and AP1 pathways, leading to cytokine release [27].

Inflammatory mediators that have been implicated in inflammation related cancer development include IL1-beta, tumor necrosis factor (TNF-alpha) and IL-6. IL1-beta and TNF-alpha induce activation of NF-kB in epithelial cells, leading to cell growth/proliferation, suppression of apoptosis, and release of other growth factors and cytokines such as EGF, IL-6, COX2 and ROS [28]. COX2 also enhances cell growth and angiogenesis, ROS modify protein function, IL-6 activates STAT3 further enhancing cell growth and stimulating growth factor production including the Reg protein [28-30]. TNF-alpha and IL-6 appear to create a positive feedback loop during cancer development, and also activate ERK/MAPK cascades known to regulate cell proliferation, migration and angiogenesis [31]. In addition, IL1-beta, TNF-alpha and IL-6 activate c-Jun kinase (JNK) resulting in cell death induced compensatory proliferation $[28,32,33]$.

\section{Role of Stem Cells in H. pylori Associated Gastric Cancer Development}

Gastric stem cells provide the capacity for renewal of all cell lineages of gastric epithelium. The molecular markers that identify epithelial stem cells in the oxyntic mucosa of the gastric body and in the antrum appear to be different, consistent with the different cellular lineages that constitute gastric glands in these two different areas of the stomach [34]. Lgr5 was shown to mark epithelial stem cells in mice and human gastric antrum [35, 36, 37•]. In the oxyntic mucosa, trefoil factor family 2 (TFF2) was reported in progenitors for mucous neck, parietal and zymogen producing cells [38]. Another marker of progenitor cells in oxyntic mucosa may be doublecortin-like kinase (Dclk1) [39]. Other putative gastric stem cell markers are ADAM17, CD44, and Musashi-1 [36, 40]. Bone marrow derived stem cells may contribute to the gastric stem cell pool in chronic gastritis and $H$. pylori associated neoplastic progression [41]. It has been postulated that the engrafted bone marrow derived stem cells may not follow a normal differentiation pathway and could undergo uncontrolled replication, progressive loss of differentiation, and neoplastic behavior [41, 42]. In recent studies, we reported that the Lgr5-positive epithelial stem cell pool is expanded in H. pylori-associated gastritis in the antrum of patients with GC. In GC patients with active H. pylori infection, Lgr5positive epithelial stem cells may be more susceptible to 
DNA damage than Lgr5-negative epithelial cells, demonstrated by increased levels of nuclear 8-hydroxydeoxyguanosine (8-OHdG), suggesting that $H$. pylori infection may contribute to GC risk by affecting epithelial stem cells in the human stomach [37•].

The potential interactions of inflammatory products or H. pylori released factors or direct bacterial interactions with stem and progenitor cells remain unknown and warrant further studies.

\section{H. pylori and Virulence Factors in Gastric Carcinogenesis}

A number of $H$. pylori virulence factors play a role in determining the patterns of disease associated with infection [8]. These virulence factors include the vacuolating cytotoxin (VacA), cytotoxin-associated antigen A (CagA) proteins, HP-NAP, oipA, and dupA [8]. The VacA toxin affects $\mathrm{T}$ and $\mathrm{B}$-lymphocytes and contributes to the ability of $H$. pylori to establish persistent chronic gastritis [43]. VacA induces ROS production and mitochondrial DNA mutation in gastric epithelial cells, while HP-NAP induces release of ROS from neutrophils [33, 44].

The CagA protein is encoded by the cagA gene, one of the genes that constitute the Cag pathogenicity island, which encodes a type IV secretion system [8]. Helicobacter pylori strains carrying a cagA gene with specific structural variants have been shown to have a stronger association with GC [8]. The CagA type $\mathrm{C}$ strains were associated with more severe degrees of atrophic gastritis and GC [45]. Helicobacter pylori strains with phosphorylation at the EPYIA site of CagA proteins are more common in East Asia than in Western countries, which may contribute to the increased incidence of GC in this region of the world [46]. There are several mechanisms by which CagA affects epithelial cells and may contribute to GC development [46]. CagA protein is injected from the bacterium into gastric epithelial cells via the type IV secretion system and then interacts with several intracellular signaling molecules in both tyrosine phosphorylation dependent and independent manners [46]. Once inside the epithelial cell CagA undergoes tyrosine phosphorylation by the epithelial cell Src protein and other signaling molecules at the EPIYA sites and binds Src homology 2 domain containing tyrosine phosphatase (SHP2), deregulating the phosphatase activity [46]. CagA-positive H. pylori induce higher levels of IL-8 and activate NF-kB, AP-1 and NFAT [47].

$\mathrm{CagA}$ was shown to interact with the hepatocyte growth factor receptor Met causing sustained activation of PI3K and Akt which leads to b-catenin and NF-kB activation [48]. CagA was found to interact with TNF receptor associated factor 6 (TRF6) and TGF-beta-activating kinase 1 (TAK1) with resulting NF-kB activation [33].

CagA related intracellular signaling potentially affects multiple cellular functions. For example, in our studies we showed that methylation of the $\mathrm{O}$ (6)-methylguanine-DNA methyltransferase (MGMT) DNA repair gene was significantly associated with CagA-positive $H$. pylori strains in chronic gastritis, suggesting a role for CagA-positive H. pylori mediated effects in epigenetic regulation [49॰]. Other effects of CagA involve interference with proteasome-mediated degradation of the tumor suppressor RUNX3 and TP53 by binding ASPP2, a modulator of P53 and gene silencing of RUNX3 [50, 51].

Studies in mice carrying a transgenic cagA gene showed gastric epithelial hyperplasia and some mice developed gastric polyps and adenocarcinomas of the stomach and small intestine [52••], further supporting a role for CagA in gastric carcinogenesis.

\section{Host Genetic Susceptibility and H. pylori Associated Gastric Cancer}

Although it is clear that development of GC is multifactorial and requires interaction with host susceptibility genetic factors, to date, only few host susceptibility factors have been confirmed, including the pro-inflammatory gene polymorphisms in IL-1beta and $I L-1 R N$ (receptor antagonist) genes which showed to increase the risk of hypochlorhydria, gastric atrophy, GC and neoplastic precursors in $H$. pylori infected patients [53].

\section{Molecular Alterations Driving H. pylori Associated Gastric Carcinogenesis}

From the early stages of $H$. pylori gastritis, the infection and associated inflammation lead to epithelial cell mutations, epigenetic, microRNA and gene expression changes, genomic instability, altered cellular signaling, and imbalanced proliferation and apoptosis of gastric epithelial cells [54], driving the progression from pre-neoplastic to neoplastic lesions [6] (Fig. 1).

Oncogene activation and loss of tumor suppressor gene function are well known mechanisms involved in development and maintenance of the cancer phenotype, and are in many instances caused by mutation or by epigenetic regulation, whether directly through hypermethylation of gene promoter regions or indirectly through epigenetic control of transcriptional or post-transcriptional regulators such as miRNAs. To establish a parallel, in cancers arising in inflammatory milieus, such as GC, mutagenesis and epigenetic deregulation are main mechanisms driving 
epithelial cells in the direction of cancer. Increased mutation burden of the epithelial genome results through two established mechanisms: 1) increased occurrence of mutations due to direct damage of DNA (free radicals, primarily ROS and NOS), that can be enhanced by aberrant expression of mutation inducing factor (AID) and 2) deficient repair of mutations prior to DNA replication (deficient DNA repair associated with reduced function of MGMT [49•] and mismatch repair (MMR) genes MLH1, MSH2 and MMR heterodimer associated proteins [55] (Fig. 1). Published data support that both mechanisms are critical for H. pylori and inflammation associated GC [33, 49•, 55]. The link between enhanced mutation burden of gastric epithelium and Helicobacter gastritis can be found at multiple levels as reviewed below.

\section{Mutations in Gastric Carcinogenesis}

The types of mutations and mechanisms of mutagenesis in gastric carcinogenesis are multiple and include microsatellite instability (MSI) resulting from altered DNA mismatch repair, point mutations and genomic instability including loss of heterozygosity (LOH), gene amplifications, rearrangements, insertion and deletion mutations, chromosomal losses and duplications. These genomic lesions accumulate during the steps of gastric carcinogenesis in cells representing intestinal metaplasia, dysplasia/ adenoma, and adenocarcinoma and probably in epithelial progenitors and stem cells [56].

Through the combined effects of $H$. pylori virulence factors and inflammatory mediators released in response to infection, ROS levels increase in the cell and lead to modification of nucleic acid bases leading to DNA damage including single or double-strand DNA breaks, DNA adducts and DNA-protein cross links [28].

Mutations of TP53 and APC genes can be detected in intestinal metaplasia and gastric dysplasia [57, 58]. TP53 mutations (in exons 5-8) characterized by G:C to A:T transitions are detected in gastric neoplasia [58]. APC mutations, including stop-codon and frameshift mutations were reported in $45 \%$ of cancers [59]. KRAS mutations in codon 12 are rare in gastric carcinogenesis and were reported in $14 \%$ of cases with atrophic gastritis, and about $10 \%$ of adenomas, dysplasias, and carcinomas [60]. Recently, the spectrum of mutations in GC has been explored by massive parallel sequencing approaches. A recent study performed whole exome sequencing in GC as compared to matching non-neoplastic tissue and determined the molecular pathways most frequently revealing gene mutations [61]. Chromatin modification and cell junction pathways showed the most significant enrichment of mutated genes. Mutations were found in members of the
SWI-SNF complex (ARIDIA, PBRMI and SMARCC1), ISWI complex (SMARCAI) and NuRD complex (CHD3, $C G D 4$ and $M B D 2$ ), and other genes encoding histonemodifying proteins (SIRT1 and SETD2), affecting $59 \%$ of GCs [61]. Overall, $59 \%$ of GCs had mutations in genes involved in cell adhesion, including $C H D 1$. Genes involved in cell cycle regulation including TP53, PTEN and TTK were mutated in $77 \%$ of GCs. Other signaling pathways frequently mutated in GCs included the Wnt-BMP-TGFbeta, axon guidance, MAPK, DNA replication, focal adhesion, ERBB, ATR-BRCA and Rb pathways [61]. Another study also using exome sequencing reported that cell adhesion was the pathway most enriched for mutations [62•]. TP53 was mutated in $66.7 \%$, and PIK3CA and ARIDIIA were mutated in $20 \%$ of GCs [62 $]$. Frequent mutations in chromatin remodeling genes (ARIDIIA, MLL3 and $M L L)$ were found in $47 \%$ of GCs [62 - . ARIDIIA mutations where associated with PIK3CA mutations and microsatellite instability [62•]. Importantly, some mutations identified in the advanced cancer stages, may have occurred early during initiation associated with $H$. pylori infection (such as MSI [15]) whereas other mutations occur late in cancer progression and are independent of $H$. pylori infection or inflammation induced by the bacterium.

As summarized in Fig. 1, the mechanisms through which $H$. pylori-associated gastritis results in enhanced mutagenesis and cellular deregulation are multiple. The increased DNA damage of epithelial cells is due to oxidative stress caused by reactive oxygen species (ROS), and reactive nitrogen species (RNS) generated by inflammatory cells as well as by gastric epithelial cells after activation by H. pylori [63]. In addition to the increased production of ROS, there is limited availability of oxygen radical scavengers, such as glutathione and gluthatione-S-transferase during $H$. pylori gastritis, which may contribute to higher levels of oxygen radicals in the mucosa of infected patients [64]. Gastric mucosa with H. pylori gastritis, intestinal metaplasia and gastric atrophy as pre-neoplastic lesions, was shown to have increased levels of DNA $8 \mathrm{OHdG}$, a marker for oxidative DNA damage [65]. Mutations associated with oxidative damage include point mutations in genes such as the tumor suppressor TP53, KRAS, and other genes involved in gastric carcinogenesis [58]. Epithelial expression of the activation-induced cytidine deaminase (AID) in $H$. pylori gastritis may induce $\mathrm{C} / \mathrm{G}$ to T/A transitions by its cytidine deaminase activity [28].

Specific deficiencies of DNA repair functions during $H$. pylori gastritis also contribute to $H$. pylori associated mutagenesis $[6,49 \bullet, 66,67]$. Altered DNA repair mechanisms include those involved in DNA MMR as well as other proteins that primarily repair DNA lesions induced by oxidative and nitrosative stress, such as MGMT and polymorphic glycosylase (OGG1). DNA MMR proteins 
(MLH1, MSH2, MSH6 and PMS2) are required for the repair of DNA replication associated sequence errors. Several studies have reported a role for DNA MMR deficiency in mutation accumulation during $H$. pylori infection $[15,37 \bullet, 49 \bullet, 55,66]$. DNA mismatch repair deficiency leads to frameshift mutagenesis that can alter the coding region of genes, as well as repetitive regions known as short tandem repeats or microsatellite regions, with resulting microsatellite instability [68]. Microsatellite instability can be detected in chronic gastritis and intestinal metaplasia from patients with GC, indicating that MSI can occur in pre-neoplastic mucosa $[15,56]$. For example, a study of microsatellite instability in the stepwise gastritis cancer sequence reported MSI in chronic gastritis (13\% of cases), intestinal metaplasia (20\% of cases), dysplasias ( $25 \%$ of cases) and GCs (38\% of cases), consistent with a role for DNA MMR deficiency in H. pylori associated gastric carcinogenesis [69]. Using a co-culture in vitro system, gastric cell lines exposed to $H$. pylori expressed reduced levels of DNA mismatch repair proteins MLH1 and MSH2 [55], and these changes were associated with increased mutagenesis of a reporter vector, including MSItype frameshift mutations as well as point mutations [66]. These studies provided definitive evidence that $H$. pylori bacteria lead to mutations in the epithelial cell genome, a hallmark of cancer.

High-level MSI (MSI-H) is associated with loss of expression and promoter hypermethylation of the MLH1 DNA mismatch repair gene in gastric adenomas and cancers. MSI has been reported in 17-35\% of gastric adenomas $[70,71]$, and in $17-59 \%$ gastric carcinomas $[15$, 70-74]. Gastric cancers with MSI-H may carry frameshift mutations that may affect the function of cancer related genes, such as BAX, IGFRII, TGFBRII, MSH3 and MSH6 [75-79].

Other DNA repair proteins are involved in the correction of oxidative stress associated mutations during $H$. pylori infection such as repair of 8-OHdG by polymorphic glycosylase (OGG1). A gene polymorphism that may affect the function of OGG1 was reported frequently in patients with intestinal metaplasia and GC, suggesting that deficient OGG1 function may contribute to increased mutagenesis during gastric carcinogenesis [80]. The DNA repair protein MGMT can remove O (6)alkylG DNA adducts. In the absence of functional MGMT these adducts are not removed and mispair with $\mathrm{T}$ during DNA replication, resulting in G-to-A mutations. MGMT-promoter methylation has been reported in various stages of gastric carcinogenesis, suggesting a role for this DNA repair protein in GC development [81]. Hypermethylation of the MGMT gene and reduced levels of MGMT proteins in the gastric epithelium, particularly in patients infected with CagApositive strains occur during $H$. pylori gastritis [49॰].
Further, in our studies, MGMT promoter methylation was shown to be partially reversible after eradication of H. pylori infection [49॰]. Overall, our studies indicate that MGMT-dependent DNA repair is disrupted during H. pylori gastritis, likely contributing to higher levels of mutagenesis in $H$. pylori infected gastric mucosa [49॰].

The nucleotide alterations introduced by $8 \mathrm{OHdG}$, a marker of oxidative DNA stress, are G/C to T/A transversions, and in earlier studies we demonstrated that these were the most common type of point mutations induced by $H$. pylori in a co-culture setting [66], thus indicating that oxidative stress similar to that induced by inflammatory mediators can be replicated by $H$. pylori interacting with epithelial cells even in the absence of inflammation. If DNA repair is effective, the mismatch $\mathrm{U}: \mathrm{G}$ caused by the $8 \mathrm{OHG}$ modification would be recognized by uracyl-DNA glycosylase or MSH2/MSH6 heterodimer and repaired. If DNA repair is not effective then these mutations are expected to accumulate, consistent with our studies showing impaired DNA repair associated with $H$. pylori infection [49•, 66, 67, 82]. In addition, other studies have shown that enhanced and aberrant activity of activation-induced cytidine deaminase (AID) in gastric epithelium in the setting of $H$. pylori infection can lead to increased U:G mismatches further contributing to the number of unrepaired mismatches and increased T/A transitions [28] (Fig. 1).

Gene targets of AID enhanced mutagenesis in gastric cells include $T P 53, C D K N-2 B-C D K N-2 A$ (encoding p16, p15 and p14 suppressor proteins), causing submicroscopic deletions with chromosome copy number losses involving the $C D K N-2 B-C D K N-2 A$ locus $[28,83]$.

\section{Epigenetic Gene Regulation and H. pylori Associated Gastric Carcinogenesis}

Epigenetic DNA modifications are inherited upon somatic cell replication and encompass $\mathrm{CpG}$ methylation and histone modifications. Methylation of $\mathrm{CpG}$ islands in promoter regions causes silencing of the downstream gene, whereas methylation within the coding region of a gene usually is associated with increased gene transcription. Cancers display regional hypermethylation of promoter regions and global hypomethylation. The extensive epigenetic alteration in the background mucosa that gives rise to dysplasia and cancers represents an epigenetic field defect in inflammation and infection associated cancers. $\mathrm{CpG}$ methylation occurs early in gastric carcinogenesis, affecting genes such as $M L H 1, \mathrm{p} 14, \mathrm{p} 15, \mathrm{p} 16$, E-cadherin, RUNX3, thromobospondin-1 (THBS1), tissue inhibitor of metalloproteinase 3 (TIMP-3), COX-2, and MGMT [6, 16, 84-88]. Methylation of these and a number of other genes is associated with chronic inflammation in the gastric 
mucosa [89]. Pro-inflammatory interleukin-1-beta polymorphisms were shown to be associated with $\mathrm{CpG}$ island methylation of target genes such as the E-cadherin gene [90]. CpG methylation of the gastric mucosa has been shown to be partially reversible after eradication of $H$. pylori infection, supporting the notion that $H$. pylori and inflammatory mediators interfere with cellular mechanisms governing epigenetic regulation in gastric epithelium $[17,49 \bullet, 91]$.

\section{Role of MicroRNAs in H. pylori Associated Gastric Carcinogenesis}

MicroRNAs have been shown to be involved in H. pylori associated diseases [92]. Increased expression of miR-21 was detected in $H$. pylori-infected gastric mucosa, as compared to noninfected tissue [93•], while $m i R-218-2$ and miR7 were downregulated [94, 95]. MicroR-204 downregulation was involved in aberrant Ras activation in gastric carcinogenesis [96]. MiR-155 may function as a negative regulator that helps fine-tune the inflammatory response to $H$. pylori infection [97].

\section{Conclusions}

New insights into the mechanisms through which $H$. pylori and mucosal inflammation lead to GC development have been unraveled in recent years. H. pylori virulence factors, in particular specific genotypes of CagA and VacA genes are important in carcinogenesis. Additional evidence has shown the role of chronic $H$. pylori infection in deregulation of epigenetic and microRNA molecular patterns and massively parallel sequencing has enabled the mutational mapping of GCs, pointing to possible new candidate genes that may be evaluated next for a driver role in $H$. pylori and inflammation associated carcinogenesis. Mutation of the epithelial cell genome, a hallmark of cancer, has been demonstrated to accumulate in the gastric epithelium of $H$. pylori infected patients, through mechanisms that include active mutation burden in a milieu contaminated with oxygen radicals and the inability of adequate DNA repair that occurs during $H$. pylori infection. Stem cells of the stomach have been shown to be targets of oxidative damage in the Helicobacter-inflammatory environment. Future research taking advantage of massively parallel sequencing genomic technologies, integrated systems biology and computational approaches are promising new tools to advance our understanding of how $H$. pylori and the consequent inflammation ultimately induce cancer in a sub-population of infected patients. Molecular markers that can identify patients with a history of $H$. pylori infection and associate with significant risk of development of GC remain a major goal of research, as are predictive markers for GC prognosis, targeted therapies, and recurrence. Identification of potential combinatorial biomarkers based on the bacterial genotypes, inflammation, and host genetic and phenotypic profiles should provide much needed tools for screening, prevention, and treatment of GC and precursor lesions.

Disclosure No potential conflicts of interest relevant to this article were reported.

\section{References}

Papers of particular interest, published recently, have been highlighted as:

- Of importance

•• Of major importance

1. Parkin DM, Bray F, Ferlay J et al (2005) Global cancer statistics, 2002. CA Cancer J Clin 55:74-108

2. Warren JR, Marshall B (1983) Unidentified curved bacilli on gastric epithelium in active chronic gastritis. Lancet 1:1273-1275

3. Herrera V, Parsonnet J (2009) Helicobacter pylori and gastric adenocarcinoma. Clin Microbiol Infect 15:971-976

4. International Agency for Research of Cancer (1994) Shistosomes, liver flukes and Helicobacter pylori. IARC Monogr Eval Carcinog Risks Hum 61:177-241

5. Palli D (2000) Epidemiology of gastric cancer: an evaluation of available evidence. J Gastroenterol 35(Suppl 12):84-89

6. Gologan A, Graham DY, Sepulveda AR (2005) Molecular Markers in Helicobacter pylori-Associated Gastric Carcinogenesis. Clin Lab Med 25:197-222

7. Sepulveda AR, Aisner D (2009) Molecular basis of diseases of the gastrointestinal tract. In: Coleman WB, Tsongalis GJ (eds) Molecular pathology: the molecular basis of human disease. Academic Press, New York, pp 365-393

8. Yamaoka Y (2012) Pathogenesis of helicobacter pylori-related gastroduodenal diseases from molecular epidemiological studies. Gastroenterol Res Pract 2012:371503

9. Sepulveda A, Goyal A (2011) Helicobacter and Gastric Neoplasms. In: Tan D, Lauwers G (eds) Advances in Surgical Pathology: Gastric Cancer. Lippincott Williams and Wilkins, Philadelphia, pp 22-37

10. Makola D, Peura DA, Crowe SE (2007) Helicobacter pylori infection and related gastrointestinal diseases. J Clin Gastroenterol 41:548-558

11. Bonifazi M, Malvezzi M, Bertuccio P et al (2011) Age-periodcohort analysis of oral cancer mortality in Europe: the end of an epidemic? Oral Oncol 47:400-407

12. Asaka M, Sugiyama T, Nobuta A et al (2001) Atrophic gastritis and intestinal metaplasia in Japan: results of a large multicenter study. Helicobacter 6:294-299

13. Recavarren-Arce S, Leon-Barua R, Cok J et al (1991) Helicobacter pylori and progressive gastric pathology that predisposes to gastric cancer. Scand J Gastroenterol 26(Suppl 181):51-57

14. Sepulveda A, Peterson LE, Shelton J et al (2002) Histological patterns of gastritis in H. pylori-infected individuals with a family history of gastric cancer. Am J Gastroenterol 97:1365-1370 
15. Leung WK, Kim JJ, Kim JG et al (2000) Microsatellite instability in gastric intestinal metaplasia in patients with and without gastric cancer. Am J Pathol 156:537-543

16. Kang GH, Shim YH, Jung HY et al (2001) CpG island methylation in premalignant stages of gastric carcinoma. Cancer Res 61:2847-2851

17. Sepulveda AR, Jones D, Ogino S et al (2009) CpG methylation analysis-current status of clinical assays and potential applications in molecular diagnostics: a report of the Association for Molecular Pathology. J Mol Diagn 11:266-278

18. Mikata R, Yokosuka O, Fukai K et al (2006) Analysis of genes upregulated by the demethylating agent 5-aza-2'-deoxycytidine in gastric cancer cell lines. Int J Cancer 119:1616-1622

19. Huang JQ, Sridhar S, Chen Y et al (1998) Meta-analysis of the relationship between Helicobacter pylori seropositivity and gastric cancer. Gastroenterology 114:1169-1179

20. Sepulveda AR, Wu L, Ota $\mathrm{H}$ et al (2000) Molecular identification of main cellular lineages as a tool for the classification of gastric cancer. Hum Pathol 31:566-574

21. Robinson K, Argent RH, Atherton JC (2007) The inflammatory and immune response to Helicobacter pylori infection. Best Pract Res Clin Gastroenterol 21:237-259

22. McGee DJ, Mobley HL (2000) Pathogenesis of Helicobacter pylori infection. Curr Opin Gastroenterol 16:24-31

23. Beigier-Bompadre M, Moos V, Belogolova E et al (2011) Modulation of the CD4 + T-cell response by Helicobacter pylori depends on known virulence factors and bacterial cholesterol and cholesterol alpha-glucoside content. J Infect Dis 204:1339-1348

24. Khamri W, Walker MM, Clark P et al (2010) Helicobacter pylori stimulates dendritic cells to induce interleukin-17 expression from CD4 + T lymphocytes. Infect Immun 78:845-853

25. - Kao JY, Zhang M, Miller MJ, et al. (2010) Helicobacter pylori immune escape is mediated by dendritic cell-induced Treg skewing and Th17 suppression in mice. Gastroenterol 138:1046-1054. This study showed that H. pylori directs Treg skewing through dendritic cell activation and inhibits Th17 immunity, allowing the chronic persistence of $H$. pylori infection which is critical for gastric carcinogenesis

26. Lamb A, Chen LF (2010) The many roads traveled by Helicobacter pylori to NFkappaB activation. Gut Microbes 1:109-113

27. Allison CC, Kufer TA, Kremmer E et al (2009) Helicobacter pylori induces MAPK phosphorylation and AP-1 activation via a NOD1-dependent mechanism. J Immunol 183:8099-8109

28. Chiba T, Marusawa H, Ushijima T (2012) Inflammation-associated cancer development in digestive organs: mechanisms and roles for genetic and epigenetic modulation. Gastroenterology 143:550-563

29. Ziech D, Franco R, Pappa A et al (2011) Reactive oxygen species (ROS)-induced genetic and epigenetic alterations in human carcinogenesis. Mutat Res 711:167-173

30. Sekikawa A, Fukui H, Fujii S et al (2008) REG Ialpha protein mediates an anti-apoptotic effect of STAT3 signaling in gastric cancer cells. Carcinogenesis 29:76-83

31. Kanda K, Komekado H, Sawabu T et al (2012) Nardilysin and ADAM proteases promote gastric cancer cell growth by activating intrinsic cytokine signalling via enhanced ectodomain shedding of TNF-alpha. EMBO Mol Med 4:396-411

32. Chen F (2012) JNK-induced apoptosis, compensatory growth, and cancer stem cells. Cancer Res 72:379-386

33. Lamb A, Chen LF (2012) Role of the Helicobacter pylori-induced inflammatory response in the development of gastric cancer. J Cell Biochem. doi:10.1002/jcb.24389

34. Mills JC, Shivdasani RA (2011) Gastric epithelial stem cells. Gastroenterology 140:412-424
35. Barker N, Huch M, Kujala P et al (2010) Lgr5(+ve) stem cells drive self-renewal in the stomach and build long-lived gastric units in vitro. Cell Stem Cell 6:25-36

36. Simon E, Petke D, Boger C et al (2012) The spatial distribution of LGR5 + cells correlates with gastric cancer progression. PLoS One $7:$ :e35486

37. - Uehara T, Ma D, Yao Y, et al. (2012) H. pylori infection is associated with DNA damage of Lgr5-positive epithelial stem cells in the stomach of patients with gastric cancer. Dig Dis Sci. [Epub ahead of print]. This study for the fist time showed that gastric epithelail stem cells are targets of mutagenesis in the H. pylori inflammatory mileau. The Lgr5-positive epithelial stem cell pool was expanded in $H$. pylori-associated gastritis in the antrum of patients with GC. In GC patients with active H. pylori infection, Lgr5-positive epithelial stem cells may be more susceptible to DNA damage than Lgr5-negative epithelial cells, suggesting that $H$. pylori infection may contribute to GC risk by affecting epithelial stem cells in the human stomach

38. Quante M, Marrache F, Goldenring JR et al (2010) TFF2 mRNA transcript expression marks a gland progenitor cell of the gastric oxyntic mucosa. Gastroenterology 139:2018-2027. e2

39. May R, Sureban SM, Hoang N et al (2009) Doublecortin and CaM kinase-like-1 and leucine-rich-repeat-containing G-proteincoupled receptor mark quiescent and cycling intestinal stem cells, respectively. Stem Cells 27:2571-2579

40. Qiao XT, Gumucio DL (2011) Current molecular markers for gastric progenitor cells and gastric cancer stem cells. J Gastroenterol 46:855-865

41. Correa P, Houghton J (2007) Carcinogenesis of Helicobacter pylori. Gastroenterology 133:659-672

42. Giannakis M, Chen SL, Karam SM et al (2008) Helicobacter pylori evolution during progression from chronic atrophic gastritis to gastric cancer and its impact on gastric stem cells. Proc Natl Acad Sci USA 105:4358-4363

43. Torres VJ, VanCompernolle SE, Sundrud MS et al (2007) Helicobacter pylori vacuolating cytotoxin inhibits activation-induced proliferation of human T and B lymphocyte subsets. J Immunol 179:5433-5440

44. Huang XW, Luo RH, Zhao Q et al (2011) Helicobacter pylori induces mitochondrial DNA mutation and reactive oxygen species level in AGS cells. Int J Med Sci 8:56-67

45. Yamaoka Y, Kodama T, Kashima K et al (1998) Variants of the $3^{\prime}$ region of the cagA gene in Helicobacter pylori isolates from patients with different $\mathrm{H}$. pylori-associated diseases. J Clin Microbiol 36:2258-2263

46. Handa O, Naito Y, Yoshikawa T (2007) CagA protein of Helicobacter pylori: a hijacker of gastric epithelial cell signaling. Biochem Pharmacol 73:1697-1702

47. Backert S, Naumann M (2010) What a disorder: proinflammatory signaling pathways induced by Helicobacter pylori. Trends Microbiol 18:479-486

48. Suzuki M, Mimuro H, Kiga K et al (2009) Helicobacter pylori CagA phosphorylation-independent function in epithelial proliferation and inflammation. Cell Host Microbe 5:23-34

49. - Sepulveda AR, Yao Y, Yan W, et al. (2010) CpG methylation and reduced expression of O6-methylguanine DNA methyltransferase is associated with Helicobacter pylori infection. Gastroenterology 138:1836-1844. This study highights the role of epigenetic regulation of the DNA repair protein MGMT in $H$. pylori infection underlying innefficent DNA repair in infected gastric mucosa which can contribute to the enhanced burden of tumorigenic mutational events in gastric epithelium during H. pylori-associated carinogenesis

50. Buti L, Spooner E, Van der Veen AG et al (2011) Helicobacter pylori cytotoxin-associated gene A (CagA) subverts the 
apoptosis-stimulating protein of p53 (ASPP2) tumor suppressor pathway of the host. Proc Natl Acad Sci USA 108:9238-9243

51. Tsang YH, Lamb A, Chen LF (2011) New insights into the inactivation of gastric tumor suppressor RUNX3: the role of $\mathrm{H}$. pylori infection. J Cell Biochem 112:381-386

52. • Ohnishi N, Yuasa H, Tanaka S, et al. (2008) Transgenic expression of Helicobacter pylori CagA induces gastrointestinal and hematopoietic neoplasms in mouse. Proc Natl Acad Sci USA 105:1003-1008. This study for the first time demonstrated an oncogenic activity of $\mathrm{H}$. pylori virulence factor CagA in vivo

53. El-Omar EM, Carrington M, Chow WH et al (2000) Interleukin-1 polymorphisms associated with increased risk of gastric cancer. Nature 404:398-402

54. Chattopadhyay R, Bhattacharyya A, Crowe SE (2010) Dual regulation by apurinic/apyrimidinic endonuclease-1 inhibits gastric epithelial cell apoptosis during Helicobacter pylori infection. Cancer Res 70:2799-2808

55. Kim JJ, Tao H, Carloni E et al (2002) Helicobacter pylori impairs DNA mismatch repair in gastric epithelial cells. Gastroenterology 123:542-553

56. Kobayashi K, Okamoto T, Takayama S et al (2000) Genetic instability in intestinal metaplasia is a frequent event leading to well-differentiated early adenocarcinoma of the stomach. Eur $\mathbf{J}$ Cancer 36:1113-1119

57. Nakatsuru S, Yanagisawa A, Furukawa Y et al (1993) Somatic mutations of the APC gene in precancerous lesion of the stomach. Hum Mol Genet 2:1463-1465

58. Shiao YH, Rugge M, Correa P et al (1994) p53 alteration in gastric precancerous lesions. Am J Pathol 144:511-517

59. Lee HS, Choi SI, Lee HK et al (2002) Distinct clinical features and outcomes of gastric cancers with microsatellite instability. Mod Pathol 15:632-640

60. Hunt JD, Mera R, Strimas A et al (2001) KRAS mutations are not predictive for progression of preneoplastic gastric lesions. Cancer Epidemiol Biomarkers Prev 10:79-80

61. Wang K, Kan J, Yuen ST et al (2011) Exome sequencing identifies frequent mutation of ARID1A in molecular subtypes of gastric cancer. Nat Genet 43:1219-1223

62. - Zang ZJ, Cutcutache I, Poon SL, et al. (2012) Exome sequencing of gastric adenocarcinoma identifies recurrent somatic mutations in cell adhesion and chromatin remodeling genes. Nat Genet 44:570-574. This study illustrates the power of massively parallel sequencing technologies to identify key tumorigenic events in gastric cancers. Application to pre-neoplastic tissue analysis promises to unravel drivers of gastric carcinogenesis that may relate to H. pylori, inflammation or other specific underlying factors

63. Shimada T, Watanabe N, Hiraishi Het al (1999) Redox regulation of interleukin-8 expression in MKN28 cells. Dig Dis Sci 44:266-273

64. Verhulst ML, van Oijen AH, Roelofs HM et al (2000) Antral glutathione concentration and glutathione S-transferase activity in patients with and without Helicobacter pylori. Dig Dis Sci 45:629-632

65. Farinati F, Cardin R, Degan P et al (1998) Oxidative DNA damage accumulation in gastric carcinogenesis. Gut 42:351-356

66. Yao Y, Tao H, Park DI et al (2006) Demonstration and characterization of mutations induced by Helicobacter pylori organisms in Gastric Epithelial Cells. Helicobacter 11:272-286

67. Park DI, Park SH, Kim SH et al (2005) Effect of Helicobacter pylori infection on the expression of DNA mismatch repair protein. Helicobacter 10:179-184

68. Umar A (2004) Lynch syndrome (HNPCC) and microsatellite instability. Dis Markers 20:179-180

69. Ling XL, Fang DC, Wang RQ et al (2004) Mitochondrial microsatellite instability in gastric cancer and its precancerous lesions. World J Gastroenterol 10:800-803
70. Kashiwagi K, Watanabe M, Ezaki T et al (2000) Clinical usefulness of microsatellite instability for the prediction of gastric adenoma or adenocarcinoma in patients with chronic gastritis. $\mathrm{Br}$ J Cancer 82:1814-1818

71. Abraham SC, Park SJ, Lee JH et al (2003) Genetic alterations in gastric adenomas of intestinal and foveolar phenotypes. Mod Pathol 16:786-795

72. Lee JH, Abraham SC, Kim HS et al (2002) Inverse relationship between APC gene mutation in gastric adenomas and development of adenocarcinoma. Am J Pathol 161:611-618

73. Hayden JD, Martin IG, Cawkwell L et al (1998) The role of microsatellite instability in gastric carcinoma. Gut 42:300-303

74. Sepulveda AR, Santos AC, Yamaoka Y et al (1999) Marked differences in the frequency of microsatellite instability in gastric cancer from different countries. Am J Gastroenterol 94:3034-3038

75. Ottini L, Palli D, Falchetti M et al (1997) Microsatellite instability in gastric cancer is associated with tumor location and family history in a high-risk population from Tuscany. Cancer Res 57:4523-4529

76. Yamamoto H, Sawai H, Perucho M (1997) Frameshift somatic mutations in gastrointestinal cancer of the microsatellite mutator phenotype. Cancer Res 57:4420-4426

77. Shinmura K, Tani M, Isogaki J et al (1998) RER phenotype and its associated mutations in familial gastric cancer. Carcinogenesis 19:247-251

78. Myeroff LL, Parsons R, Kim SJ et al (1995) A transforming growth factor beta receptor type II gene mutation common in colon and gastric but rare in endometrial cancers with microsatellite instability. Cancer Res 55:5545-5547

79. Chung YJ, Park SW, Song JM et al (1997) Evidence of genetic progression in human gastric carcinomas with microsatellite instability. Oncogene 15:1719-1726

80. Farinati F, Cardin R, Cassaro M et al (2008) Helicobacter pylori, inflammation, oxidative damage and gastric cancer: a morphological, biological and molecular pathway. Eur J Cancer Prev 17:195-200

81. Park TJ, Han SU, Cho YK et al (2001) Methylation of O(6)methylguanine-DNA methyltransferase gene is associated significantly with K-ras mutation, lymph node invasion, tumor staging, and disease free survival in patients with gastric carcinoma. Cancer 92:2760-2768

82. Kim J, Leung W, Wu L et al (2000) Helicobacter pylori impairs DNA mismatch repair in gastric epithelial cells: a link with gastric carcinogenesis. Gastroenterology 118:A867

83. Matsumoto Y, Marusawa H, Kinoshita K et al (2010) Up-regulation of activation-induced cytidine deaminase causes genetic aberrations at the CDKN2b-CDKN2a in gastric cancer. Gastroenterology 139:1984-1994

84. Toyota M, Ahuja N, Suzuki H et al (1999) Aberrant methylation in gastric cancer associated with the $\mathrm{CpG}$ island methylator phenotype. Cancer Res 59:5438-5442

85. To KF, Leung WK, Lee TL et al (2002) Promoter hypermethylation of tumor-related genes in gastric intestinal metaplasia of patients with and without gastric cancer. Int J Cancer 102:623-628

86. Waki T, Tamura G, Sato M et al (2003) Promoter methylation status of DAP-kinase and RUNX3 genes in neoplastic and nonneoplastic gastric epithelia. Cancer Sci 94:360-364

87. Kang GH, Lee S, Kim JS et al (2003) Profile of aberrant CpG island methylation along the multistep pathway of gastric carcinogenesis. Lab Invest 83:635-641

88. Lee JH, Park SJ, Abraham SC et al (2004) Frequent CpG island methylation in precursor lesions and early gastric adenocarcinomas. Oncogene 23:4646-4654

89. Kang GH, Lee HJ, Hwang KS et al (2003) Aberrant CpG island hypermethylation of chronic gastritis, in relation to aging, gender, 
intestinal metaplasia, and chronic inflammation. Am J Pathol 163:1551-1556

90. Qian X, Huang C, Cho $\mathrm{CH}$ et al (2008) E-cadherin promoter hypermethylation induced by interleukin-1beta treatment or H. pylori infection in human gastric cancer cell lines. Cancer Lett 263:107-113

91. Chan AO, Peng JZ, Lam SK et al (2006) Eradication of Helicobacter pylori infection reverses E-cadherin promoter hypermethylation. Gut 55:463-468

92. Belair C, Darfeuille F, Staedel C (2009) Helicobacter pylori and gastric cancer: possible role of microRNAs in this intimate relationship. Clin Microbiol Infect 15:806-812

93. - Zhang Z, Li Z, Gao C, et al. (2008) miR-21 plays a pivotal role in gastric cancer pathogenesis and progression. Lab Invest 88:1358-1366. This study began to elucidate the potential contribution of microRNAs in H. pylori associated carcinogenesis, showing that miR-21 was significantly overexpressed in H. pyloriinfected gastric mucosa, and implying that overexpression of miR-21 in gastric cancer may be due in part to $H$. pylori infection

94. Volinia S, Calin GA, Liu CG et al (2006) A microRNA expression signature of human solid tumors defines cancer gene targets. Proc Natl Acad Sci USA 103:2257-2261

95. Kong D, Piao YS, Yamashita $S$ et al (2012) Inflammationinduced repression of tumor suppressor miR-7 in gastric tumor cells. Oncogene 31:3949-3960

96. Lam EK, Wang X, Shin VY et al (2011) A microRNA contribution to aberrant Ras activation in gastric cancer. Am J Transl Res 3:209-218

97. Xiao B, Liu Z, Li BS et al (2009) Induction of microRNA-155 during Helicobacter pylori infection and its negative regulatory role in the inflammatory response. J Infect Dis 200:916-925 